\title{
The use of musculoskeletal ultrasound in a Rheumatology Outpatient Clinic
}

\author{
Annamaria Iagnocco, Fulvia Ceccarelli, Carlo Perricone, Angelica Gattamelata, \\ Annacarla Finucci, Eleonora Ricci, Chiara Scirocco, Iolanda Maria Rutigliano, \\ Vasilia Iorgoveanu, Elena Cravotto, Guido Valesini
}

Rheumatology Unit, Dipartimento Medicina Interna e Specialità Mediche, Sapienza Università di Roma, Rome, Italy

\begin{abstract}
Introduction: Musculoskeletal ultrasound (US) represents a valid, reliable and sensitive-to-change tool for the evaluation of patients suffering from rheumatic conditions. This method demonstrates a wide applicability and availability, finding place in the clinical practice in rheumatology outpatient clinic. Aim: To perform an epidemiological evaluation related to the use of US in a university rheumatology outpatient clinic. Material and methods: During a 3-month period, data concerning consecutive patients attending to the US Unit of Department of Rheumatology, Sapienza University of Rome were registered. We collected the demographic data, the diagnosis, the reason for the US examination, the examined joints, as well as the requesting physicians' specialty. Results: In the October-December 2013 period, 572 patients (M/F 137/435; mean age \pm SD $55.2 \pm 15.8$ years) were registered. The US examination was more frequently requested for the following diseases: rheumatoid arthritis (29.5\%), osteoarthritis (10.6\%), spondyloarthritis (9.1\%), and connective tissue diseases (8.9\%). In 239 of cases (41.8\%), the US evaluation was requested for other indications. The US evaluation was requested slightly more frequently for monitoring $(55.7 \%)$ compared to diagnosis (44.3\%). The requesting physician was a rheumatologist in the majority of the cases $(80.6 \%)$. The most frequent requested were the hand joints $(28.9 \%)$ and wrists $(23.3 \%)$. Conclusions: US examinations are most frequently used in the evaluation of patients with rheumatoid arthritis and mainly to monitor the disease. The exam is requested mostly by rheumatologists. The hand joints and wrists were the most frequently evaluated.
\end{abstract}

Abstract

Keywords: ultrasound, rheumatology, clinical applications

\section{Introduction}

An early and accurate diagnosis and careful management of the patients affected by rheumatic diseases is a priority of the rheumatologist. Clinical examination cannot differentiate between joint and peri-articular pathology, making difficult the achievement of precise diagnosis $[1,2]$. Moreover, the coexistence of both inflammatory and degenerative lesions could be another difficulty for the physician.

Received 20.09.2014 Accepted 10.10.2014

Med Ultrason

2014, Vol. 16, No 4, 332-335

Corresponding author: Annamaria Iagnocco,

Dipartimento Medicina Interna e Specialità

Mediche: Reumatologia,

Sapienza Università di Roma,

V.le del Policlinico 155, Rome - 00161, Italy.

Phone: +3906 49974634

Fax: +3906 49974642

Email: annamaria.iagnocco@uniroma1.it
The application of musculoskeletal ultrasound (MSUS) in rheumatology has rapidly evolved and this technique has been incorporated into routine clinical practice over the past decade. The diffusion of MS-US is largely the result of the technological development that has been allowed the production of high quality and easily interpretable images. The interest of the rheumatologists was particularly focused on the inflammatory arthritis and the early identification of structural damage [3-6].

Various rheumatic conditions require US examination both for the disease diagnosis and the monitoring. Given this large variability of diseases assessed with US, an epidemiological analysis of the MS-US use in the clinical practice could be interesting. In the light of these premises, in the present study we aimed to perform an epidemiological description related to the use of US in a university rheumatology outpatient clinic. 


\section{Material and methods}

We performed an epidemiological evaluation in a 3-months period by including consecutive patients who attended Ultrasound Unit of Department of Rheumatology, Sapienza University of Rome. The patients were referred to our Unit for different musculoskeletal manifestations. Informed consent was obtained from all patients. We collected from all patients the following information: demographic data (age, sex), diagnosis, reason for performing the US examination (diagnostic or monitoring), requesting physicians' specialty, and joints examined.

US examination of the requested areas was performed by the rheumatologists expert on MS-US from our department. The study was performed with a MyLab70 XVG (Esaote, Italy) machine equipped with a multifrequency linear probe $(4-13 \mathrm{MHz}, 6-18 \mathrm{MHz})$ in grey scale and power Doppler mode. US examination was carried out according to European League Against Rheumatism (EULAR) guidelines for each anatomic region; the OMERACT definitions was applied to identify the US abnormalities $[7,8]$. All the identified findings were recorded in a standardized US report.

\section{Statistical analysis}

We used version 13.0 of the SPSS statistical package. Normally distributed variables were summarized using the mean $\pm \mathrm{SD}$, and non-normally distributed variables by the median and range. The frequencies were summarized as percentage. Univariate comparisons between nominal variables were calculated using chi-square test or Fisher-test where appropriate. Twotailed $\mathrm{P}$ values were reported, $\mathrm{P}$ values less than 0.05 were considered significant.

\section{Results}

We study a cohort of 572 patients (F/M 435/137; mean age \pm SD $55.2 \pm 15.8$ years).

Table I reports the frequency of the indications for the US examination that has been required mostly in patients affected by rheumatoid arthritis (RA) (169 cases, 29.5\%).

Within the connective tissue disease, 17 patients were affected by systemic lupus erythematosus (SLE), 16 by undifferentiated connective tissue disease, 15 by Sjogren's syndrome (SS), and finally 3 by systemic sclerosis $(\mathrm{SSc})$.

Table I. Indications for the US examination

\begin{tabular}{lc}
\hline Indication & $\mathbf{N}(\mathbf{\%})$ \\
\hline Rheumatoid Arthritis & $169(29.5)$ \\
Osteoarthritis & $61(10.6)$ \\
Spondyloarthritis & $52(9.1)$ \\
Connective Tissue Diseases & $51(8.9)$ \\
Psoriatic arthritis & $36(6.3)$ \\
Polymyalgia rheumatica & $12(2.1)$ \\
Fibromyalgia & $11(1.9)$ \\
Microcrystal arthropathies & $6(1.0)$ \\
Other conditions & $174(30.4)$ \\
\hline
\end{tabular}

Table II. Results on the joint examined with ultrasound.

\begin{tabular}{lc}
\hline Examined joint & N (\%) \\
\hline Hand joints & $403(28.9)$ \\
Wrist & $325(23.3)$ \\
Knee & $193(13.8)$ \\
Feet joints & $190(13.6)$ \\
Ankle & $94(6.7)$ \\
Shoulder & $86(6.2)$ \\
Elbow & $57(4.1)$ \\
Hip & $46(3.3)$ \\
\hline
\end{tabular}

Table III. Results of assessed joints according to the pathology

\begin{tabular}{lcccccccc}
\hline & $\begin{array}{c}\text { Shoulder } \\
\mathbf{N}(\%)\end{array}$ & $\begin{array}{c}\text { Elbow } \\
\mathbf{N}(\%)\end{array}$ & $\begin{array}{c}\text { Hand } \\
\mathbf{N}(\%)\end{array}$ & $\begin{array}{c}\text { Wrist } \\
\mathbf{N}(\%)\end{array}$ & $\begin{array}{c}\text { Hip } \\
\mathbf{N}(\%)\end{array}$ & $\begin{array}{c}\text { Knee } \\
\mathbf{N}(\%)\end{array}$ & $\begin{array}{c}\text { Ankle } \\
\mathbf{N}(\%)\end{array}$ & $\begin{array}{c}\text { Feet } \\
\mathbf{N}(\%)\end{array}$ \\
\hline RA & $13(2.9)$ & $13(2.9)$ & $141(32.1)$ & $125(28.5)$ & $7(1.6)$ & $53(12.1)$ & $35(7.9)$ & $82(18.6)$ \\
OA & $8(7.2)$ & $1(0.9)$ & $37(33.3)$ & $22(19,8)$ & $8(7.2)$ & $23(20.7)$ & $2(1.8)$ & $10(9.0)$ \\
AS & $6(4.3)$ & $5(3.6)$ & $44(31.8)$ & $29(21.0)$ & $3(2.2)$ & $16(11.6)$ & $11(7.9)$ & $24(17.4)$ \\
SLE & $1(2.9)$ & - & $12(35.3)$ & $8(23.5)$ & - & $6(17.6)$ & - & $7(20.6)$ \\
SS & $1(3.2)$ & - & $12(38.7)$ & $11(35.5)$ & $2(6.5)$ & $1(3.2)$ & $1(3.2)$ & $3(9.6)$ \\
UCTD & - & $4(13.8)$ & $10(34.5)$ & $9(31.0)$ & $1(3.4)$ & $3(10.3)$ & $2(6.9)$ & - \\
SSc & - & - & $3(50.0)$ & $2(33.3)$ & - &. & - & $1(16.6)$ \\
PsA & $7(6.7)$ & $10(9.6)$ & $24(23.1)$ & $20(19.2)$ & $1(0.9)$ & $18(17.3)$ & $8(7.7)$ & $16(15.4)$ \\
PR & $5(21.7)$ & - & $5(21.7)$ & $3(13.0)$ & $4(17.4)$ & $2(8.7)$ & $2(8.7)$ & $2(8.7)$ \\
FM & $4(12.5)$ & $1(3.1)$ & $10(31.2)$ & $5(15.6)$ & $1(3.1)$ & $5(15.6)$ & $1(3.1)$ & $5(15.6)$ \\
CP & - & - & - & $1(11.1)$ & - & $4(44.4)$ & $2(22.2)$ & $2(22.2)$ \\
\hline
\end{tabular}

Legend: RA: Rheumatoid Arthritis; OA: osteoarthritis; AS: ankylosing spondylitis; SLE: Systemic Lupus Erythematosus; SS: Sjögren Syndrome; UCTD: undifferentiated connective tissue diseases; SSc: Systemic Sclerosis; PsA: Psoriatic Arthritis; PR: Polymialgia Rheumatica; FM: Fibromyalgia; CP: Crystal pathologies. 
Table IV. Reason for US examination.

\begin{tabular}{lcc}
\hline Disease & $\begin{array}{c}\text { Monitoring } \\
\mathbf{N}(\%)\end{array}$ & $\begin{array}{c}\text { Diagnosis } \\
\mathbf{N}(\%)\end{array}$ \\
\hline RA & $128(75.7)$ & $41(24.3)$ \\
OA & $30(49.1)$ & $31(50.8)$ \\
SA & $30(57.7)$ & $22(42.3)$ \\
SLE & $13(76.4)$ & $4(23.5)$ \\
SS & $10(62.5)$ & $6(37.5)$ \\
UCTD & $11(68.7)$ & $5(31.2)$ \\
SSc & $2(66.6)$ & $1(33.3)$ \\
PsA & $20(55.5)$ & $16(44.4)$ \\
PR & $4(33.3)$ & $8(66.6)$ \\
FM & $5(45.4)$ & $6(54.5)$ \\
CP & $4(66.6)$ & $2(33.3)$ \\
\hline
\end{tabular}

Legend: RA: Rheumatoid Arthritis; OA: osteoarthritis; AS: ankylosing spondylitis; SLE: Systemic Lupus Erythematosus; SS: Sjögren Syndrome; UCTD: undifferentiated connective tissue diseases; SSc: Systemic Sclerosis; PsA: Psoriatic Arthritis; PR: Polymialgia Rheumatica; FM: Fibromyalgia; CP: Crystal pathologies.

Concerning the joints evaluated the most frequent requested were the hand joints (403 cases, $28.9 \%$ ), followed by wrists $(325,23.3 \%)$, and knees (193 cases, $33.7 \%$ ). Table II reports the frequency of the joints examined by US.

The relative frequencies of the assessed joints depending of the underlying disease are detailed in table III.

In $\mathrm{OA}$ the most frequently examined joints were the hand and the knee. The most frequently scanned joints in polymialgia rheumatica (PR) were the shoulders ( 5 cases, $21.7 \%$ ) and hands (5 cases, 21.7\%). In fibromyalgia hand joints were mostly assessed whereas in microcrystal arthropathy (gout and chondrocalcinosis) was the knee. In 174 of cases (30.4\%) the US exam was requested for other indications.

At the full sample level, the US evaluation was requested more frequently for monitoring (55.7\%) compared to diagnosis (44.3\%). Table IV reports the results on this aspect in the different diseases.

The physician requesting the US examination was a rheumatologist in the majority of cases (461 cases, $80.6 \%$ ), with a statistical significant difference compared to the general practitioner (21 cases, $3.7 \%$; $<<0.0001$ ), the orthopedic surgeon ( 3 cases, $0.5 \% ; \mathrm{p}<0.0001$ ) or other physicians ( 87 cases, $15.2 \%$; $\mathrm{p}<0.0001$ ).

\section{Discussions}

The present study represents an epidemiological analysis that has been conducted to better characterize the use of MS-US in a university rheumatology unit.

In a period of three months follow-up, more than 500 patients with different rheumatic conditions were evaluated by using US: this first and simple data confirms the diffusion of this technique in the clinical rheumatology practice.

The results, indicating that RA was the most frequent indication for US assessment, underline the great interest of rheumatologists for this condition: data from the literature indicate the growing interest on the US application in patients affected by RA [9-13]. Thanks to its great sensitivity in detecting abnormalities since early phase of disease and in identifying subclinical synovitis, US assessment is recommended as a complementary tool to the clinical evaluation [14]. In our opinion the results concerning the use of US in the patients affected by CTD such as SLE are of particular interest, as they confirm that US is used in different rheumatic pathologies other than arthropathies. The hand and wrist were the most evaluated joints, followed by knee; these results show that the identification of RA is the most frequent indication for US examination. As widely demonstrated, these joints are the more frequently involved in patients affected by RA, but also in other rheumatic conditions, such as CTD or psoriatic arthritis. As expected, considering the joint involvement of the disease, the shoulder were more frequently evaluated in patients with PR.

In our patients the US examination was requested especially to monitor the rheumatic diseases except in patients with PR. In this group of patients, the US was requested mostly for the disease diagnosis $(66.6 \%)$; this result is not surprising when considering the difficulties of clinical examination in the evaluation of shoulder and hip, the most frequently involved joints in PR.

Finally, another important aspect is that almost exclusively rheumatologists required the US evaluation. This finding may be interpreted in the context of the increasing applications and use of US in the rheumatology clinical practice with a growing interest of rheumatology in the field.

In conclusion, US is a feasible imaging tool that has registered increasing applications in the rheumatology clinical practice over the last years. The presence of an US unit that is integrated in a university rheumatology department improves the applications of US in different rheumatic diseases as well as in different contexts. These aspects further increase the use of MS-US in rheumatology in the concept of US as a bedside procedure that should be routinely applied in the daily practice in rheumatology.

\section{Conflict of interest: none}

\section{References}

1. Grassi W, Filippucci E, Carotti M, Salaffi F. Imaging modalities for identifying the origin of regional musculoskeletal pain. Best Pract Res Clin Rheumatol 2003; 17:17-32. 
2. Kang T, Lanni S, Nam J, Emery P, Wakefield RJ. The evolution of ultrasound in rheumatology. Ther Adv Musculoskelet Dis 2012; 4:399-411.

3. Naredo E, Aguado P, De Miguel E, et al. Painful shoulder: comparison of physical examination and ultrasonographic findings. Ann Rheum Dis 2002; 61:132-6.

4. Meenagh G, Iagnocco A, Filippucci E, et al. Ultrasound imaging for the rheumatologist IV: ultrasonography of the knee. Clin Exp Rheumatol 2006; 24: 35-60.

5. Iagnocco A, Filippucci E, Meenagh G, et al. Ultrasound imaging for the rheumatologist I: ultrasonography of the shoulder. Clin Exp Rheumatol 2006; 24:6-11.

6. Riente L, Delle Sedie A, Iagnocco A, et al. Ultrasound imaging of the rheumatologist V: ultrasonography of the ankle and foot. Clin Exp Rheumatol 2006; 24: 493-498.

7. Backhaus M, Burmester GR, Gerber T, et al. Guidelines for musculoskeletal ultrasound in rheumatology - Working Group for Musculoskeletal Ultrasound in the EULAR Standing Committee on International Clinical Studies including Therapeutic Trials. Ann Rheum Dis 2001; 60: 641-649.

8. Wakefield RJ, Balint PV, Szkudlarek M, et al. OMERACT 7 Special Interest Group. Musculoskeletal ultrasound in- cluding definitions for ultrasonographic pathology. J Rheumatol 2005; 32: 2485-2487.

9. Rizzo C, Ceccarelli F, Gattamelata A et al. Ultrasound in rheumatoid arthritis. Med Ultrason 2013; 15: 199-208.

10. Botar-Jid C, Bolboaca S, Fodor D et al. Gray scale and power Doppler ultrasonography in evaluation of early rheumatoid arthritis. Med Ultrason 2010; 12: 300-305.

11. Iagnocco A, Naredo E, Wakefield $\mathrm{R}$ et al. Responsiveness in rheumatoid arthritis. a report from the OMERACT 11 ultrasound workshop. J Rheumatol 2014; 41: 379-382.

12. Wakefield RJ, D'Agostino MA, Naredo E et al. After treatto-target: can a targeted ultrasound initiative improve RA outcomes? Ann Rheum Dis 2012; 71: 799-803.

13. Filippucci E, Iagnocco A, Meenagh G et al. Ultrasound imaging for the rheumatologist VII. Ultrasound imaging in rheumatoid arthritis. Clin Exp Rheumatol 2007; 25: $5-10$.

14. Mandl P, Kurucz R, Niedermayer D, Balint PV, Smolen JS. Contributions of ultrasound beyond clinical data in assessing inflammatory disease activity in rheumatoid arthritis: current insights and future prospects. Rheumatology (Oxford) 2014, doi: 10.1136/annrheumdis-2014-205585. 